investigación económica, vol. LXVIII, número especial 2009, pp. 15-20

\title{
Introducción del Editor
}

Ignacio Perrotini Hernández*

La búsqueda y elaboración de reglas monetarias racionales con el propósito de conseguir los objetivos de estabilidad macroeconómica es de larga data, y se remonta por lo menos a los orígenes de la ciencia económica. Adam Smith afirma en su Riqueza de las Naciones que "el papel moneda bien regulado [puede sustituir a la circulación de metales] no sólo sin ningún inconveniente, sino, en algunos casos, con algunas ventajas" (1776, libro iv, i.). ${ }^{1}$

Con el fin de conseguir la estabilidad macroeconómica, Nueva Zelanda adoptó un modelo de objetivo de inflación (OI) como marco de política monetaria en 1990. Desde entonces, un número creciente de países industrializados, emergentes y en desarrollo han abrazado este nuevo régimen cuyo objetivo de política prístino es alcanzar y mantener tasas de inflación

\footnotetext{
* Facultad de Economía de la Universidad Nacional Autónoma de México (UNAm), <iph@servidor. unam. $m x>$.

${ }^{1}$ David Ricardo, Henry Thornton, la Banking School, la Currency School, Walter Bagehot y Knut Wicksell discutieron la relevancia de las reglas monetarias en el siglo XIX, mientras que en el siglo Xx Henry Simons y Milton Friedman, entre otros, antecedieron a John Taylor. El caso de Wicksell (1898 y 1922) es notable porque se anticipó a Taylor (1993), Ball (1998) y Svensson (2000) al postular el papel de la tasa de interés como reguladora de los precios en una economía con tipo de cambio flexible.
} 
bajas y estables (Bernanke et al. 1999; Taylor 1999; Woodford 2003). La preeminencia global de la estrategia or la documenta, además, el hecho de que el marco de política monetaria actual de principalísimos bancos centrales como la Reserva Federal de Estados Unidos (Fed), el Banco Central Europeo, el Banco de Inglaterra y el Banco Nacional de Suiza opera sobre la base de varios elementos esenciales del régimen or (Blanchard 2005; IMF 2005).

Por otra parte, en la Carnegie Rochester Conference on Public Policy de noviembre de 1992, John Taylor (1993) formuló la proposición de que la evolución de la tasa de interés de los fondos federales $(r)$ de la Fed durante el lustro previo podía representarse con una sencilla ecuación, a saber:

$$
r=p+0.5 y+0.5(p-2)
$$

donde $y$ denota la desviación porcentual del producto interno bruto real (PIB) respecto del producto potencial y $p$ es la tasa de inflación de los cuatro trimestres anteriores. Esta ecuación parsimoniosa pronto se convirtió en la famosa regla de Taylor, y es utilizada como función de reacción en los modelos macroeconómicos de los bancos centrales que operan con el régimen OI. Así, el régimen or se basa en algún tipo de regla de Taylor. Es digno de mencionar el que la naturaleza de la regla de Taylor admite la posibilidad de considerar la estabilidad de la producción como un objetivo de la política monetaria, lo cual contrasta con la praxis monetaria de los bancos centrales que enfocan su atención sólo en la inflación.

El advenimiento de la regla de Taylor ha reavivado el debate reglas versus discreción en política monetaria (Blinder 1998; Lavoie 2004). La adopción de la regla de Taylor frecuentemente ha sido el resultado de crisis de regímenes monetarios donde el tipo de cambio operaba como ancla de los precios, sobre todo en las economías emergentes. Pero el régimen oI también se ha adoptado como alternativa a la regla de Milton Friedman que postula metas de crecimiento constante de los agregados monetarios. En algunos casos, como en ciertos países de América latina y Europa Central y Oriental, el régimen or se introdujo como un elemento más de reformas económicas más amplias. Es decir, casi nunca la transición fue deliberada y suave. 
Un hecho estilizado incontestable es que en las economías emergentes el tipo de cambio desempeña un papel más relevante en la consecución de la estabilidad de precios en comparación con lo que se observa en las economías industrializadas. En general, casi en todos los casos la adopción de una regla de Taylor como marco de política monetaria siguió a episodios previos de desinflación en los que los puntos de partida fueron niveles de inflación y volatilidad de los precios mayores en los países emergentes en comparación con lo observado en los desarrollados. En los primeros, los choques de precios relativos (fluctuación del tipo de cambio, precios de la energía) y de oferta tienden a inducir desviaciones de la inflación vis-à-vis el objetivo del Banco Central.

El abandono -las más de las veces precipitado y desordenado- de anclas nominales centradas en el supuesto de una oferta monetaria exógena o en la fijación del tipo de cambio, por un lado, y la creciente adopción del régimen de metas de inflación, por otro, ha conferido a la regla de Taylor el aura de la gran innovación monetaria del presente (Arestis 2002), el talante de un nuevo consenso monetario. México, por ejemplo, inició esta transición a posteriori de la crisis financiera de 1994-1995 y acogió formalmente el régimen oi en 2001. Por esta razón, es fundamental comprender el modus operandi del nuevo régimen monetario, analizar sus virtudes y dificultades, evaluar su consistencia lógica y contrastar sus hipótesis con la evidencia empírica disponible. Así, los artículos contenidos en el presente volumen especial de Investigación Económica dedicado al análisis del régimen de metas de inflación, pretenden contribuir a este debate.

Angeriz y Arestis analizan la evidencia empírica tanto para economías industrializadas como emergentes que operan con el régimen oI y concluyen que, dado que otros países que no tienen un régimen or también han tenido éxito en disminuir y estabilizar la inflación, el régimen or no es condición sine qua non de la estabilidad de precios. Más aún, dada la presencia de choques de oferta, la excesiva concentración en la inflación puede inducir inestabilidad de la macroeconomía real y costos reales asociados a la estabilidad de la inflación. 
El artículo de Guadalupe Mántey analiza la experiencia de México; argumenta que el 'miedo a flotar' y el efecto de traspaso de las fluctuaciones cambiarias al nivel de precios, determinan que el tipo de cambio continúe desempeñando el papel de ancla nominal, paradójicamente, en un régimen de tipo de cambio flexible. Por esta razón, el Banco de México en la práctica utiliza las intervenciones esterilizadas en los mercados cambiarios como un instrumento adicional de política monetaria. Estas intervenciones implican un costo fiscal, por lo que sugiere que la política económica debería hacerse cargo de los problemas estructurales de la inflación y de la volatilidad del tipo de cambio.

Sergio Rossi explica las virtudes de un régimen or flexible como el que sigue Suiza. Esta flexibilidad permite a las autoridades monetarias suizas contrarrestar los impactos reales de choques de oferta y financieros similares a los que se han tornado más frecuentes desde el colapso de Bretton Woods y con la reciente financiarización del capitalismo global. La flexibilidad es la clave del éxito en la estabilidad macroeconómica, afirma, y la transparencia y la rendición de cuentas son factores auxiliares. Sin la necesaria flexibilidad, el régimen oI puede incluso obstruir la independencia de la política monetaria y su contribución al mejor desempeño macroeconómico.

Esteban Pérez estudia el caso del régimen or de Chile; identifica dos fases, la del or ecléctico (1991-1999) y la del oi completo (1999-presente). El hallazgo más importante de este artículo es que los países que padecen restricciones de balanza de pagos en su dinámica de crecimiento y que adoptan el régimen oI, tarde o temprano experimentarán un conflicto de intereses entre objetivos internos y objetivos externos. Dado que en general el conflicto se resuelve a favor de los objetivos externos, puede decirse que el límite que confina las bondades de la ley de Taylor es la conocida ley de Thirlwall que postula que en el largo plazo el equilibrio de la balanza de pagos determina la tasa de crecimiento máxima alcanzable. Este resultado es crucial sobre todo en las economías emergentes que se caracterizan por desempleo involuntario, tasas de sacrificio reales asociadas a la desinflación e inflación estructural. 
Ferrari y Fabris analizan el régimen or de Brasil y evalúan los principales resultados macroeconómicos registrados en el periodo 1999-2008. Sus resultados muestran que el caso brasileño se ha caracterizado por: tasas de inflación superiores a las establecidas por el objetivo; volatilidad de las (altas) tasas de interés y del tipo de cambio; efectos de traspaso del tipo de cambio a la inflación; inflación de costos asociados a choques de oferta que predominan por sobre los choques de demanda; inflación inercial; deterioro macroeconómico (crecimiento, empleo) y fragilidad de la deuda pública debido a las elevadas tasas de interés.

Finalmente, agradezco a Álvaro Angeriz, Philip Arestis, Maria Juliana Fabris, Fernando Ferrari Filho, Guadalupe Mántey de Anguiano, Esteban Pérez Caldentey y Sergio Rossi quienes con entusiasmo y diligencia aceptaron colaborar con este número especial de Investigación Económica. Agradezco también a Karina Navarrete por su esfuerzo y excelente trabajo en la formación de esta edición especial.

\section{REFERENCIAS}

Arestis, P., "Editor's Introduction", The Manchester School, vol. 70, núm. 4, Special Issue, 2002, pp. 483-486.

Ball, L., "Policy rules for open economies", National Bureau of Economic Research (NBER), Documento de Trabajo núm. 6760, 1998.

Bernanke, B.S.; T. Laubach; F.S. Mishkin y A. Posen, Inflation Targeting: Lessons from the International Experience, Princeton, Princeton University Press, 1999.

Blanchard, O., "Monetary policy and unemployment", en W. Semmler (ed.), Monetary Policy and Unemployment: The Us, Euro-area, and Japan, Londres y Nueva York, Routledge, 2005.

Blinder, A.S., Central Banking in Theory and Practice, Cambridge, The Massachusetts Institute of Technology (MIT) Press, 1998.

International Monetary Fund (IMF), World Economic Outlook, Washington, IMF, 2005. Lavoie, M., "The new consensus on monetary policy seen from a Post-Keynesian perspective", en M. Lavoie y M. Seccareccia (eds.), Central Banking in the Modern World: Alternative Perspectives, Edward Elgar, Cheltenham, UK, Northampton, MA, USA, 2004. 
Smith, A., An Inquiry into the Nature and Causes of the Wealth of Nations, Londres, 1776.

Svensson, L.E.O., "Open-economy inflation targeting”, en Journal of International Economics, vol. 50, núm. 1, 2000, pp. 155-183.

Taylor, J.B., "Discretion versus policy rules in practice", Carnegie-Rochester Conference Series on Public Policy, núm. 39, 1993, pp. 199-214.

_ (ed.), Monetary Policy Rules, Chicago y Londres, The University of Chicago Press, 1999.

Wicksell, K. (1898a), "The influence of the rate of interest on commodity prices", en E. Lindhal, (ed.), Knut Wicksell: Selected Papers on Economic Theory, Allen y Unwin, Londres, 1969, pp. 67-89.

Wicksell, K., Guldfrågan, 1922.

Woodford, M., Interest and Prices: Foundations of a Theory of Monetary Policy, Princeton, Princeton University Press, 2003. 